\title{
Surfaces
}

\section{LA TOILE FAIT-ELLE AUTORITÉ ?}

\section{Sylvain Rheault}

Volume 8, 1999

HUMANITÉS ET INFORMATIQUE : QUI A LES COMMANDES ?

HUMANITIES AND COMPUTING: WHO'S DRIVING?

URI : https://id.erudit.org/iderudit/1065077ar

DOI : https://doi.org/10.7202/1065077ar

Aller au sommaire du numéro

\section{Éditeur(s)}

Les Presses de l’Université de Montréal

ISSN

1188-2492 (imprimé)

1200-5320 (numérique)

Découvrir la revue

Citer cet article

Rheault, S. (1999). LA TOILE FAIT-ELLE AUTORITÉ ? Surfaces, 8.

https://doi.org/10.7202/1065077ar

\section{Résumé de l'article}

La mise en ligne d'informations dans les humanités peut se comparer au travail de l'encyclopédiste : accumulation et organisation. Des questions se posent et des pistes s'ouvrent avec cette comparaison. L'accumulation modulaire qu'encourage la Toile peut-elle modifier nos pratiques d'écriture, voire nos modes de pensée ? Quant à l'organisation, encore plus cruciale, elle commande aujourd'hui de repenser l'évaluation par les pairs.
Ce document est protégé par la loi sur le droit d'auteur. L'utilisation des services d'Érudit (y compris la reproduction) est assujettie à sa politique d'utilisation que vous pouvez consulter en ligne.

https://apropos.erudit.org/fr/usagers/politique-dutilisation/ 


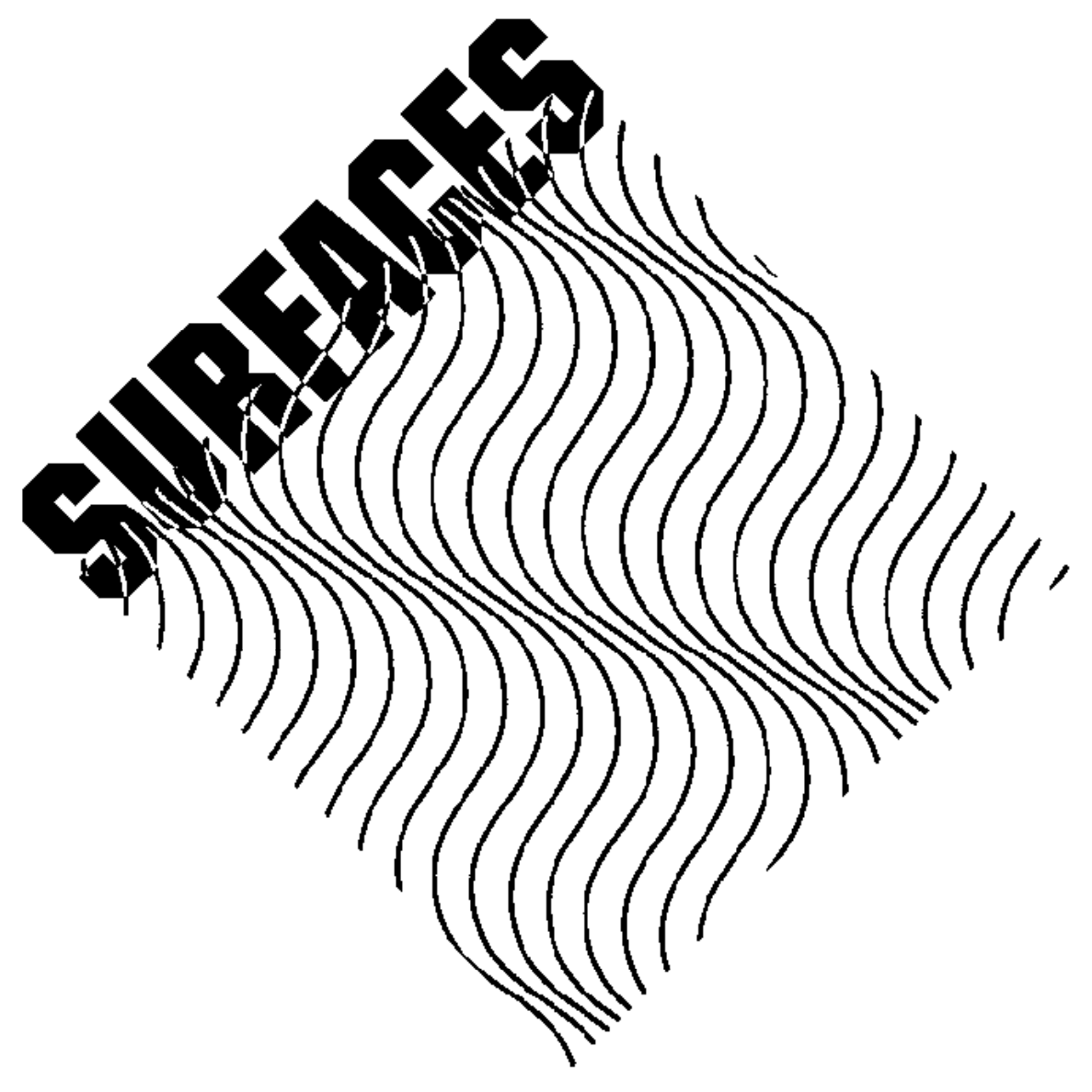

LA TOILE FAIT-ELLE AUTORITÉ ?

Sylvain Rheault 


\section{Rédacteur en chef:}

Jean-Claude Guédon

\section{Comité fondateur:}

Wlad Godzich

Walter Moser

Michel Pierssens
Christie McDonald

Antonio Gómez-Moriana

Gilles Bibeau

\section{Remerciements:}

SURFACES peut paraître grâce à un soutien des Presses de l'Université de Montréal, ainsi qu'à une aide matérielle importante (locaux, mobilier, services informatiques) de l'Université de Montréal.

\section{Adresses:}

\section{SURFACES}

Département de littérature comparée Université de Montréal C.P. 6128, succ. Centre-ville Montréal, Qc, Canada H3C 3J7

Tél.: (514) 3435683

Fax: (514) 3435684

Accès sur INTERNET www.pum.umontreal.ca/revues/surfaces

Assistance technique et questions éditoriales: guedon@ere.umontreal.ca

ISSN: $1188-2492$ 


\section{Soumission des textes}

Vous pouvez soumettre vos articles, revues, notes, commentaires ou autres aux éditeurs par courrier électronique, par disquette ou par lettre. En ce qui concerne les notes bibliographiques, veuillez suivre le protocole du MLA. Nous vous demandons également de joindre à votre texte un court résumé de 3 à 4 lignes.

Tout article publié que l'auteur voudra modifier ultérieurement sera de nouveau évalué. Si accepté, il se substituera à la première version avec une nouvelle date et la mention v. 2.

Tout texte reste la propriété de son auteur. Néanmoins, SURFACES demande d'être citée à l'occasion de toute autre publication du texte en question.

\section{Citation des textes}

Pour toute citation, veuillez consulter le document source afin d'obtenir la dernière version du texte qui vous intéresse et vous baser sur la pagination flottante (/pp. $\mathrm{xx} /$ ) plutôt que sur la foliation (pied de page). Si vous n'avez pas accès au courrier électronique, adressez-vous aux éditeurs qui pourront vous vendre une copie papier ou sur disquette. 


\title{
RÉSUMÉ
}

La mise en ligne d'informations dans les humanités peut se comparer au travail de l'encyclopédiste : accumulation et organisation. Des questions se posent et des pistes s'ouvrent avec cette comparaison. L'accumulation modulaire qu'encourage la Toile peut-elle modifier nos pratiques d'écriture, voire nos modes de pensée ? Quant à l'organisation, encore plus cruciale, elle commande aujourd'hui de repenser l'évaluation par les pairs.

\begin{abstract}
Making information in the humanities available on the Web can be compared to the work of the encyclopedist: accumulation and organization. This comparison opens up many questions and trails one might follow. Can modular accumulation, which is encouraged by the Web change our writing practices, and eventually our modes of thought? As for organization, even more crucial, it calls for a reengineering of the referereeing process.
\end{abstract}


CITATION. Passage emprunté à un auteur qui peut faire autorité. (Littré)

\section{Introduction}

L'immensité du contenu de la Toile devrait en faire une véritable encyclopédie. L'étymologie (egkuklios paideia) suggère un ouvrage faisant le tour du savoir, où sont accumulées et classées les connaissances humaines. La Toile thésaurise à un rythme exponentiel des contenus de toutes sortes, il est vrai, mais la surabondance de l'information et surtout son inorganisation peuvent nuire à la moisson d'informations utiles. L'internaute qui se branche pour la première fois découvre très vite que taper un mot trop fréquent comme patron de fouille à la page d'un moteur de recherche populaire donne une pléthore de sites à explorer. Face aux milliers de pages offertes sur la Toile, la personne intéressée aux arts ou aux sciences à la recherche d'informations pertinentes (c'est-à-dire qui méritent d'être citées dans un essai) ne sait pas toujours où donner de la tête et il est banal de rappeler que les choses empireront avec le temps. Le besoin de synthèse se fait sentir pour ne pas se noyer dans ce qui est devenu aujourd'hui un océan d'informations (Field, 1998).

On peut favoriser l'accès aux pages présentant un certain intérêt pour la recherche en restreignant l'espace virtuel à explorer. La création d'un lieu virtuel propre aux arts et sciences humaines, qui éviterait la situation maintenant classique d'avoir à trouver un électron dans une botte d'atomes, pourrait devenir un enjeu critique pour l'exploitation des nouvelles technologies.

L'encyclopédie imprimée peut être citée: il s'agit d'une référence fiable, d'un savoir dont les éléments et la structure sont reconnus de manière incontestable. Il n'en est pas de même pour la grande majorité du matériel qu'on trouve sur la Toile et c'est pourquoi le chercheur en art ou en sciences humaines citera /pp. 5-6/ de préférence un texte "réel" à un texte "virtuel" tant que les connaissances mises en ligne ne feront pas autorité. 


\section{La Toile et l'autorité}

Pour les humanités, l'autorité d'un document pourrait se définir comme le droit ou le prestige d'être cité. Cela suppose une supériorité de mérite esthétique ou scientifique qui émane soit de la qualité supérieure du travail, soit du prestige de son auteur. Ainsi:

[...] c'est seulement à partir d'un supplément à la $4 \mathrm{e}$ édition (1815-1824) qu'elle [1'Encyclopaedia Britannica] devient prestigieuse, en demandant des articles à des auteurs célèbres (James Mill, Walter Scott, Arago); dans la 13e édition, de 1926, à Einstein pour Space Time, à Freud pour Psychoanalysis, à Trotski sur Lénine. (Meschonnic, 1991, p.218)

Fonder l'autorité sur la qualité propre d'un ouvrage était une évidence aux âges où il y avait encore peu de livres. Cette époque médiatique, que Regis Debray (Debray, 1994) appelle la logosphère, était caractérisée par la tradition orale, mais aussi par l'écrit d'avant l'imprimerie. On recopiait les documents les plus intéressants. L'ère suivante, la graphospère, voyait la transmission des connaissances s'accélérer grâce au texte imprimé. L'ouvrage et son auteur n'étaient plus les seuls garants du mérite puisque l'imprimeur (ou le censeur) avait dorénavant son mot à dire dans le choix des textes à publier. La qualité du document devait compter avec la complaisance de tiers qui en garantissaient les mérites ou l'exemption de certains défauts. Aujourd'hui encore, le simple fait pour un travail d'être publié en livre, dans une collection prestigieuse, constitue une garantie de qualité. Pour les lettres françaises, par exemple, la collection de la Pléiade a établi un standard dans les éditions critiques. Nous en sommes maintenant à l'époque de la transmission de l'information par l'écran, la vidéosphère. Le savoir déjà reconnu y conserve toute son autorité. Ainsi, l'Encyclopédie de Diderot, qu'une équipe de Chicago (ARTFL) se prépare à mettre en ligne, deviendra /pp. 6-7/ une référence utile pour tous les dixhuitiémistes. La valeur intrinsèque du document mis en ligne ainsi que la qualité de l'équipe 
d'universitaires qui encadre le projet font instantanément du site une autorité sur la Toile.

Par contre, il n'en va pas de même pour la plupart des nouveaux contenus qui apparaissent chaque jour. La Toile a cet extraordinaire pouvoir de donner à toute personne munie d'un ordinateur et d'un accès à un serveur la capacité de publier à l'échelle internationale des documents virtuels. En ce sens, chacun devient le garant de son propre mérite. Mais que vaut cette caution? Si "faire autorité" signifie "posséder une valeur supérieure", dans un contexte de démocratisation de l'information (sous-entendu: "pour les bien nantis de la technologie"), puisque tous les internautes se retrouvent sur un pied d'égalité en ce qui a trait à la diffusion et à la consommation des données, le simple fait pour un document d'être affiché sur la Toile ne peut plus constituer aujourd'hui une garantie de sa qualité.

Comment alors un document affiché sur la Toile peut-il gagner le mérite qui lui permettra de faire autorité? Il suffit d'imaginer la quantité d'informations maintenant disponible pour se rendre compte qu'il serait vain d'attendre une reconnaissance des pairs, c'est-à-dire de tous les autres internautes. Rappelons que le manifeste du Unabomber (voir http://www.rectaltronics.com/ manifesto.html, entre autres), texte qu'on trouve beaucoup plus facilement sur la Toile qu'en version imprimée et qui a suscité beaucoup de commentaires, ne doit sa célébrité qu'au seul "prestige" de son auteur. Afin que la Toile devienne un instrument de savoir utile pour les arts et les sciences humaines, il faudrait accorder aux documents pertinents le mérite qui les mettra en évidence.

\section{Accumulation des connaissances et autorité}

L'accumulation des connaissances, la première démarche de la recherche, prend la forme, pour les humanités, de textes d'oeuvres (éditions critiques de préférence) et d'études, sous forme de monographies ou d'articles. On trouve maintenant de plus /pp. 7-8/ en plus de versions numérisées d'oeuvres les plus importantes, avec pas ou peu d'appareil critique. Les commentaires viendront avec le 
temps. Les chercheurs trouveront plusieurs avantages à se référer à un texte en ligne, comme le renvoi à une source unique pour tous, la facilité d'accès au document ainsi que la mise à jour continuelle des commentaires. Voilà un type de document électronique que l'on pourra citer.

En ce qui a trait aux études, l'exemple de l'île, le Centre de documentation virtuel sur la littérature québécoise, un projet récent, illustre bien le processus d'attribution de mérite. L'Île regroupe sur un seul site des informations sur les écrivains québécois et leurs oeuvres, incluant des articles que l'on peut télécharger, moyennant des frais. Ainsi sont mises à la portée des chercheurs des connaissances utiles qui font autorité non seulement parce que les articles proviennent de publications pertinentes, mais aussi parce qu'il s'agit d'un site dirigé par des universitaires.

La tendance à la publication électronique va en grandissant note Darnton (Darnton, 1999) dans un article sur le nouvel âge du livre qui soulève les nombreux problèmes auxquels doivent ou devront faire face les publications savantes. Les coupures budgétaires affectant les éditions universitaires limitent le nombre de monographies qui peuvent être publiées. Les conséquences sont fâcheuses surtout pour les nouveaux professeurs dont l'avenir académique dépend des performances de publication. Les bibliothèques, aussi victimes de coupures, restreignent leurs achats de périodiques, les périodiques, avec moins de vente, doivent alors augmenter leurs prix, ce qui grève davantage le budget des bibliothèques. Darnton constate qu'il y n'y a guère d'autres choix que d'en venir à la publication électronique. Les monographies et articles affichés sur Internet deviendraient accessibles à tous, assureraient une excellente diffusion des connaissances et cela à des coûts moindres que le support papier.

Toutes ces études pourraient former la matière de base d'une encyclopédie, à la condition première d'en garantir la qualité. C'est ici que les comités de lecture des publications savantes jouent /pp. 8-9/ un rôle important. Ce système collégial et traditionnel d'évaluation fait autorité. En guise d'argument contraire on pourrait citer le 
canular de Sokal (Sokal, 1996), à qui une publication sérieuse avait accepté une parodie d'article, mais le comité de lecture reste la meilleure instance pour évaluer le mérite d'un document. En fait, le travail d'expertise consiste simplement, dans le contexte du cyberespace, à donner ou non à un document l'aval pour figurer sur un site savant reconnu. Les études recueillies deviennent ainsi des modules de connaissances qui, ultérieurement, pourront être hiérarchisés dans une structure plus vaste que le site où ils sont affichés.

Faut-il supposer que l'information adaptée à la Toile deviendra modulaire? On sait qu'une page sur la Toile qui contient trop d'informations se voit découpée en plus petits segments, pour en faciliter la lecture à l'écran. Au site de l'as sociation des bibliothécaires universelle (voir $A B U$ dans la bibliographie), de même qu'à celui de la bibliothèque nationale de France (www.bnf.com), les oeuvres électroniques intégrales sont données une page à la fois. Lorsqu'il s'agit de citer, il est beaucoup plus pratique de référer par un lien hypertexte à un fichier ayant environ la longueur d'un article qu'à celui d'un texte tout entier. Puisque le format de l'article, module de connaissance, apparait mieux adapté à une utilisation pour la Toile, le livre, le lieu par excellence de la réflexion suivie, risque-t-il de voir son contenu se morceler? Faudra-t-il, par exemple, pour mieux préparer les penseurs de demain aux réalités de la Toile, ne plus envisager que des thèses par articles? Rappelons que la Toile est d'abord un réseau, ce qui implique, à cause de la dispersion des ressources, un morcellement de l'information. Voilà, pour le domaine des arts et des sciences humaines, un aspect à considérer sérieusement puisque la forme même du savoir risque de changer pour s'adapter au médium. La pensée sur la Toile s'organisera peutêtre en modules denses, quasi irréductibles et quasi autonomes, donc récupérables et recyclables, comme le défend William Winder (Winder, 1997). Nous ne pouvons que soulever la question, sans pouvoir y répondre pour l'instant.

/pp. 9-10/

Mais accumuler des connaissances n'est pas tout. Il faut encore les encadrer au moyen d'un système ou d'un classement. L'accumulation et l'organisation constituent les deux opérations fondatrices d'une oeuvre encyclopédique. 


\section{Organisation des connaissances et autorité}

Il faut imposer une structure aux données pertinentes disponibles sur la Toile, ne fut-ce que pour faciliter la recherche de l'information. Pour faire un parallèle historique, rappelons que le XVIIIe siècle a été celui de l'accumulation des connaissances, ces dernières ayant été organisées ensuite au XIXe siècle avec la division des sciences. Depuis, de nouvelles branches spécialisées n'ont cessé de voir le jour, permettant d'approfondir les domaines du savoir. Pour que la Toile devienne un outil de savoir, son contenu doit faire l'objet d'une organisation.

Nous avons vu plus tôt que la Toile ne hiérarchise surtout pas les contenus; toutes les pages semblent données comme ayant une valeur semblable. On peut supposer que l'aventure médiatique qu'est la Toile restera toujours égalitaire (pour ceux qui peuvent se brancher) et cette caractéristique "démocratique" nuit en quelque sorte à son organisation puisque le contenu pertinent côtoie des pages "impertinentes". Des portails comme Yahoo (www.yahoo.com) proposent bien un semblant de choix catégoriels, mais la tentative qui prétend être exhaustive reste partielle, compte tenu du volume d'informations estimé de la Toile, et partiale aussi, puisque la disposition d'un lien sur une page répond souvent à des visées commerciales. L'information y est dans un état non pas chaotique mais anarchique, puisqu'il existe tout de même une certaine organisation établie au petit bonheur des liens créés. Le contenu de la Toile représente d'abord les intérêts des personnes capables de mettre en ligne des pages de format HTML. En ce sens, l'encyclopédie que pourrait être la Toile apparaît démocratique avant d'être savante.

Une encyclopédie ne se lit pas comme une monographie; elle est divisée en articles. Il en est de même pour la Toile, /pp. 10-11/ on l'a vu, puisque les contenus, par délocalisation, sont dispersés. Les connaissances ainsi morcellées se présentent comme des données plus aisément manipulables, qu'on peut réorganiser, par exemple, selon un ordre alphabétique (qui est en fait un désordre logique). Le 
portail (comme Yahoo, Alta Vista, Excite, etc.) semble l'option la mieux adaptée à la Toile pour organiser efficacement des connaissances dispersées. D'ailleurs, la popularité d'un site comme Yahoo ne se dément pas. On consulte ce portail parce qu'il est pratique; ses contenus organisés en catégories permettent de circuler aisément d'un niveau à l'autre, de revenir si on s'est trompé de chemin.

Les portails pour les humanités restent encore, croyons-nous, des projets à réaliser. On pourrait citer Clicnet, qui est consacré à la langue française (http://swarthmore.edu/humanities/ clicnet), mais malgré son utilité incontestable, on ne peut pas dire de ce site qu'il fait autorité. Le travail d'organisation des connaissances de la Toile devrait se limiter aux contenus dont le mérite est déjà confirmé et serait confié à un "comité du portail" qui aura non plus pour tâche d'évaluer les connaissances destinées à figurer sur les sites plus prestigieux de la Toile mais de classifier celles qui font déjà autorité dans des catégories créées selon les besoins des humanités. Le comité se porte garant de la scientificité du travail de classement et de hiérarchisation du portail. Ce lien premier, offrira non seulement un classement mais aussi une démarche pour circuler dans les connaissances. Un tel programme pourrait s'inspirer, par exemple, du système de classement "Library of Congress" en usage dans la plupart des bibliothèques universitaires nord-américaines. Mais toute démarche d'organisation sérieuse serait à encourager afin de multiplier les approches du savoir. Ces stratégies permettraient, entre autres choses, de rapprocher les sciences, et de tendre à la "consilience" prônée par E. O. Field (Field, 1998).

\section{Conclusion}

La Toile, réseau dont les données dispersées et morcellées forment une masse chaotique de données, ne constitue pas (encore) une encyclopédie pour les sciences humaines et ce /pp. 11-12/ pour deux raisons. À l'accumulation des connaissances il manque l'organisation. Les contenus pertinents existent mais trop souvent manquent les mécaniques de légitimation. Pourtant, avec son contenu, ses liens et ses portails, la Toile possède un potentiel 
indiscutable pour l'accumulation et la classification. Dans l'histoire des idées, les encyclopédies ont permis de faire le point sur les connaissances et de donner un nouvel élan à l'exploration des domaines encore en friche. Avec ses stocks gigantesques de connaissances, la Toile promet la réalisation de grands avancements.

Pourquoi ne pas s'appuyer sur le prestige des comités de lecture pour désigner les connaissances pertinentes et réserver ainsi un espace virtuel propre aux sciences humaines? Ce serait en tout cas un moyen d'échapper aux fouilles pléthoriques ou inconsistantes. S'amorce du même coup une solution au problème de classification: il devient possible d'organiser des modules de connaissances limités en nombre et au mérite déjà reconnu.

L'action patiente d'attribution du mérite par les comités de lecture et de sélection de liens contribuera, à coups de tri et de structuration, à transformer les connaissances accumulées sur la Toile en savoir.

\section{Sylvain Rheault Department of French University of Regina sr@cafe.edu}

\section{Liste des sources}

$A B U$ : la Bibliothèque universelle (http://cedric.cnam.fr/)

Ce site propose "l'accès libre au texte intégral d'oeuvres du domaine public francophone sur Internet".

ARTFL Project (http://humanities.uchicago.edu/ARTFL/)

Le projet "American and French Research on the Treasury of the French Language" a numérisé des textes en /pp. 12-13/ français totalisant plus de 150 millions de mots. Ce site a été une source précieuse pour de nombreuses recherches, plus particulièrement dans le domaine de la lexicologie. 
Brandt, Scott D. 1997. "Constructivism: teaching for understanding the Internet", Communications of the ACM, vol.40 no10, $112-116$

Darnton, Robert. 1999. "The New Age of the Book", The New York Review of Books, March 18

Debray, R_gis. 1994. Manifestes medialogiques, Paris, Gallimard

Encyclopédie ou dictionnaire raisonné des sciences des arts et des métiers

(http://humanities.uchicago.edu/ARTFL/projects/encyc/overvi ew.html)

La célèbre encyclopédie du XVIIIe siècle dirigée par Denis Diderot est en voie d'être numérisée à l'Université de Chicago. Avec les moyens de fouilles qui seront mis à la disposition des chercheurs, ce site sera certainement cité dans des centaines, voir des milliers d'articles à venir.

Field, Edward O. 1998. Consilience, New York, Alfred A. Knopf

L'Île: Le Centre de documentation virtuel sur la littérature québécoise

(http://www.litterature.org)

Ce site offre des informations sur les écrivains québécois et leurs oeuvres ainsi que des articles.

Klein, Julie Thomson et Newell, William H., "Interdisciplinary Studies into the 21st Century", Journal of General Education, vol.45 no2 (1996), 152-173

/pp. 13-14/

McCarty, Willard. 1998. What Is Humanities Computing? Toward a Definition of the Field. (Texte d'une conférence prononcée au printemps 1998) http://ilex.cc.kcl.ac.uk/ wlm/essays/what 
McNeil, Tony et Mansfield, Charlie, "Baudelaire for Net Surfers: French studies and the Internet", French Studies Bulletin, 1997, Summer 63, p.10-12

Meschonnic, Henri. 1991. Des mots et des mondes: dictionnaires, encyclopédies, grammaires, nomenclatures, Paris, Hatier

Pelikan, Jaroslav. 1992. The Idea of a University: a Re-examination, New Haven: Yale University Press.

Rey, Alain. 1982. Encyclopédies et dictionnaires, Paris, Presses Universitaires de France

Rischard, Jean-François. 1996. "Connecting Developing Countries to the Information Technology Revolution", SAIS Review, $16.1,93-107$

Rodowick, D. N. 1995. "Audiovisual Culture and Interdisciplinary Knowledge", New Literary History, vol.26 no1, 111-121

Sills, David J. 1996. "Integrative Thinking, Synthesis and Creativity in Interdisciplinary Studies" Journal of General Education, vol.45 no2, 129-151

Sokal, Alan. 1996. "Transgressing the boundaries: Toward a Transformative Hermeneutics of Quantum Gravity, Social Text, no46/47, 217-252

Voir le site www.physics.nyu.edu/faculty/sokal/ à propos des conséquences de son canular.

/pp. 14-15/

Winder, William. 1997. "Texpert Systems" in Computing in the Humanities Working Papers http://www.epas.utoronto.ca: 8080/epc/chwp/winder2/

/p. 15/ 